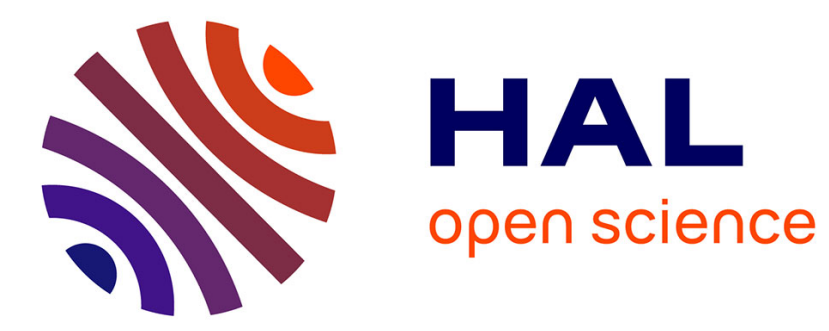

\title{
Strained Bottlebrushes in Super-Soft Physical Networks
}

Charles Clair, Abdelaziz Lallam, Martin Rosenthal, Michael Sztucki, Mohammad Vatankhah-Varnosfaderani, Andrew N Keith, Yidan Cong, Heyi Liang, Andrey V Dobrynin, Sergei S Sheiko, et al.

\section{- To cite this version:}

Charles Clair, Abdelaziz Lallam, Martin Rosenthal, Michael Sztucki, Mohammad VatankhahVarnosfaderani, et al.. Strained Bottlebrushes in Super-Soft Physical Networks. ACS Macro Letters, 2019, 8 (5), pp.530-534. 10.1021/acsmacrolett.9b00106 . hal-02415156

\author{
HAL Id: hal-02415156 \\ https://hal.science/hal-02415156
}

Submitted on 7 Jan 2021

HAL is a multi-disciplinary open access archive for the deposit and dissemination of scientific research documents, whether they are published or not. The documents may come from teaching and research institutions in France or abroad, or from public or private research centers.
L'archive ouverte pluridisciplinaire HAL, est destinée au dépôt et à la diffusion de documents scientifiques de niveau recherche, publiés ou non, émanant des établissements d'enseignement et de recherche français ou étrangers, des laboratoires publics ou privés. 


\title{
Strained Bottlebrushes in Super-Soft Physical Networks
}

\author{
Charles Clair, ${ }^{1}$ Abdelaziz Lallam, ${ }^{1}$ Martin Rosenthal, ${ }^{2}$ Michael Sztucki, ${ }^{2}$ Mohammad Vatankhah-Var- \\ nosfaderani, ${ }^{3}$ Andrew N. Keith, ${ }^{3}$ Yidan Cong, ${ }^{3}$ Heyi Liang, ${ }^{4}$ Andrey V. Dobrynin, ${ }^{4}$ Sergei S. Sheiko, ${ }^{3,5}$
} and Dimitri A. Ivanov*6,7,8,9

1 Laboratoire de Physique et Mécanique Textiles, F-68093 Mulhouse Cedex, France;

2 European Synchrotron Radiation Facility, F-38043 Grenoble, France;

3 Department of Chemistry, University of North Carolina at Chapel Hill, Chapel Hill, NC 27599, USA;

4 Department of Polymer Science, University of Akron, Akron, OH, 44325-3909, USA;

5 Institute of Macromolecular Compounds of the Russian Academy of Sciences, St. Petersburg, 199004, Russian Federation;

6 Institut de Sciences des Matériaux de Mulhouse-IS2M, CNRS UMR 7361, F-68057 Mulhouse, France;

7 Lomonosov Moscow State University, Faculty of Fundamental Physical and Chemical Engineering, Leninskie Gory 1/51, 119991 Moscow, Russian Federation;

8 Moscow Institute of Physics and Technology (State University), Institutskiy per. 9, Dolgoprudny, Russian Federation;

9 Institute of Problems of Chemical Physics, Russian Academy of Sciences, Chernogolovka, Moscow region, 142432, Russian Federation.

KEYWORDS: Bottlebrush Copolymer, Small-Angle X-ray Scattering, Microphase Separation, Aggregation Number.

\begin{abstract}
ABA triblock copolymers composed of a poly(dimethylsiloxane) (PDMS) bottlebrush central block and linear poly(methyl methacrylate) (PMMA) terminal blocks self-assemble into a physical network of PDMS bottlebrush strands connected by PMMA spherical domains. A combination of Small- and Ultra-Small-Angle X-ray scattering techniques was used to concurrently examine dimensions of PMMA spherical domains and PDMS bottlebrush strands both in the bulk and at the PMMA-PDMS interface. In agreement with scaling model predictions, the degrees of polymerization of the bottlebrush backbone $\left(n_{b b}\right)$ and PMMA block $\left(n_{A}\right)$ correlate with the measured PMMA domain size and area per molecule at the PMMA-PDMS interface as $D_{A} \propto\left(n_{b b} n_{A}\right)^{1 / 3}$ and S $\propto$ $n_{A}^{2 / 3} n_{b b}^{-1 / 3}$, respectively. In the bulk, bottlebrush strands are extended due to steric repulsion between the side chains and unfavorable interactions between the different blocks. At the PMMA-PDMS interface with large curvature, packing constraints could require additional bottlebrush backbone extension and alignment of side chains along the backbone in the direction perpendicular to the interface.
\end{abstract}

Brush-like polymers enable the design of super-soft and hyperelastic elastomers, in which side chains grafted onto network strands act as both entanglement diluents and mechanical property regulators.[1] Precise and independent tuning of side-chain degree of polymerization and grafting density yields materials with Young's moduli down to $\sim 10^{2} \mathrm{~Pa}$ and elongation-at-break up to 10.[2] These low moduli are typically achieved in swollen networks such as hydrogels, yet brush-like elastomers require no solvent and thus are immune from property degradation, caused by solvent evaporation or leakage upon deformation.

While chemically crosslinked bottlebrush networks replicate the modulus of polymeric gels and biological tissues, they fail to recreate tissue's extreme strain-stiffening behavior.[2] For instance, the Young's modulus of soft tissues such as brain and lung $\left(\sim 10^{2}-10^{3} \mathrm{~Pa}\right)$ is rapidly followed by twothree orders of magnitude increase during deformation. To enhance material strain-stiffening, we designed physical networks that self-assemble from ABA-triblock copolymers with chemically and physically distinct linear A-blocks and bottlebrush B-blocks (Figure 1a).[3] As a result of strong segregation of the blocks, at rest, network strands are stretched beyond what is observed in chemically crosslinked elastomers leading to significant enhancement of the strainstiffening behavior. Although the overall extension of polymer chains in block-copolymer systems is well established,[4] the backbone conformation inside the cylindrical envelope of bottlebrush strands remains unknown. In contrast to our previous publications [1-3], the present Letter is focused on elucidating the plastomer morphology (strand conformation).

To characterize conformation of bottlebrush blocks in the microphase-separated ABA networks, we employed a combination of Small- and Ultra-Small Angle X-ray scattering 
(SAXS and USAXS) that span length scales from Angstroms to micrometers. For this study, a series of ABA triblock copolymers with systematically varied degrees of polymerization (DP) of a central poly(dimethylsiloxane) (PDMS)-based bottlebrush block (B) $\left(n_{b b}\right)$ and poly(methyl methacrylate) (PMMA) linear terminal blocks $(\mathbf{A})\left(n_{A}\right)$ was synthesized (Table 1). Each monomeric unit of the bottlebrush backbone carries one PDMS side chain with an effective DP of $n_{s c}=14$ determined as $n_{s c}=M_{s c} / M_{0}$, where $M_{s c}=1024 \mathrm{~g} / \mathrm{mol}$ and $M_{0}=74 \mathrm{~g} / \mathrm{mol}$ are molar masses of macromonomer and PDMS monomer, respectively.

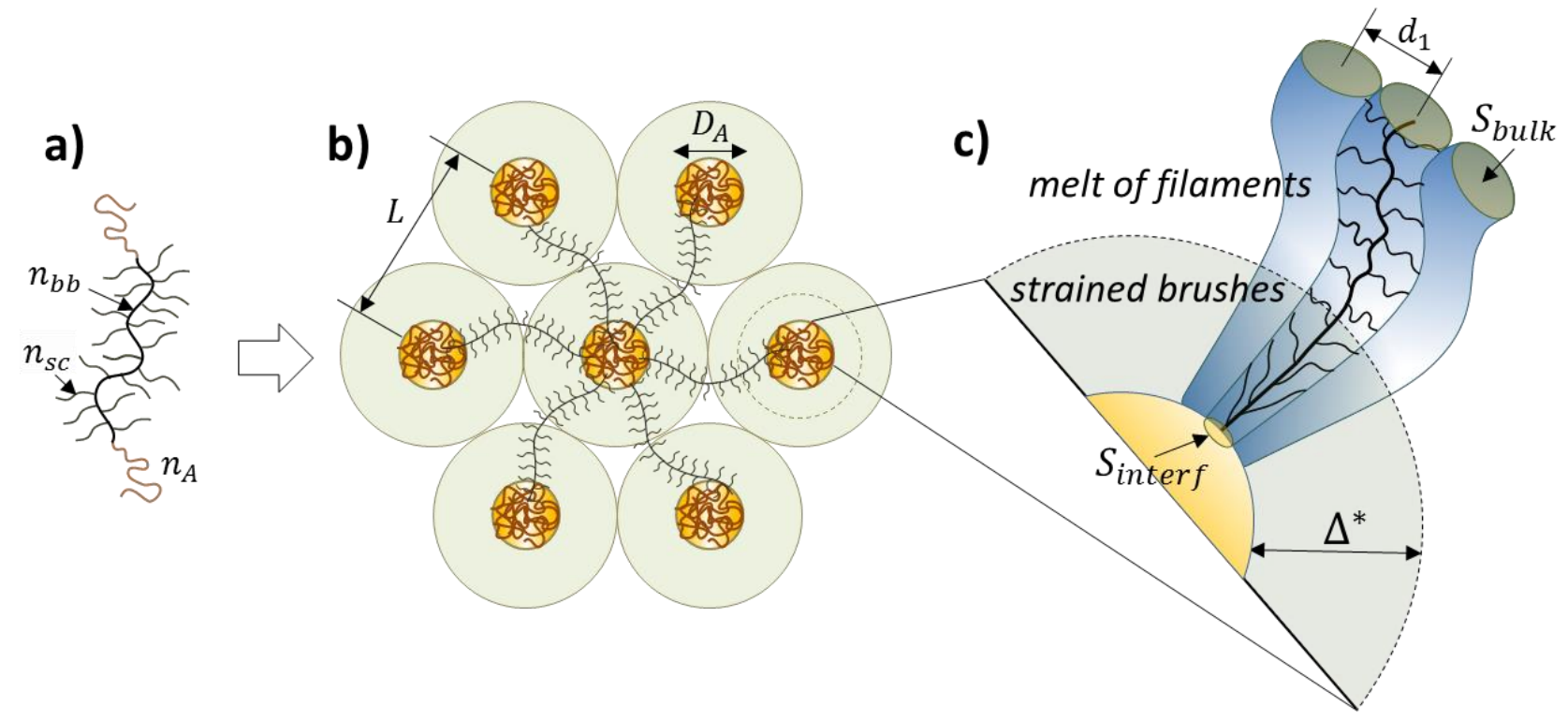

Figure 1. a): Microphase separation of (a) ABA bottlebrush copolymers results in formation of a (b) physical network composed of hard PMMA spheres imbedded in a soft matrix of PDMS bottlebrush blocks. Hexagonal packing of PMMA hard spheres covered with soft PDMS shells represents an idealized structure (see text for details). Longer PMMA-blocks may result in non-spherical morphologies [3] that are not discussed in this paper. (c) Schematics representing the conformation of the bottlebrush block anchored on a spherical PMMA domain. For a strongly curved interface the cross-section of the bottlebrush block increases with distance from the PMMA domain surface from $S_{\text {interf }}$ to $S_{\text {bulk. }}$

Table 1: Parameters of the ABA (linear-bottlebrush-linear) systems.

\begin{tabular}{|c|c|c|c|c|c|c|c|c|}
\hline Sample ${ }^{1)}$ & $\mathbf{n}_{\mathrm{bb}}{ }^{2)}$ & $n_{A}{ }^{2)}$ & $\phi_{\mathrm{A}, \mathrm{ch}}{ }^{4)}$ & $\left.\mathrm{D}_{\mathrm{A}},{ }^{5}\right) \mathrm{nm}$ & RSD ${ }^{6)}$ & $\begin{array}{l}d_{3},{ }^{7)} \\
\text { nm }\end{array}$ & $Q^{8)}$ & $\begin{array}{c}S_{\text {interf }}{ }{ }^{9)} \\
\mathbf{n m}^{2}\end{array}$ \\
\hline 300_1 & 302 & 100 & 0.051 & 20.8 & 0.12 & 42.9 & 333 & 4.1 \\
\hline $300 \_2$ & 302 & 166 & 0.082 & 25.5 & 0.11 & 45.0 & 373 & 5.5 \\
\hline 300_3 & 302 & 276 & 0.130 & 30.2 & 0.10 & 45.7 & 372 & 7.7 \\
\hline 900_1 & 938 & 190 & 0.032 & 24.3 & 0.12 & 55.5 & 280 & 6.6 \\
\hline 900_2 & 938 & 325 & 0.053 & 32.3 & 0.09 & 59.3 & 386 & 8.5 \\
\hline 900_3 & 938 & 656 & 0.102 & 50.9 & 0.11 & 78.8 & 749 & 10.9 \\
\hline 900_4 & 938 & 1235 & 0.177 & 1 & I & 141.0 & I & I \\
\hline 1200_1 & 1065 & 360 & 0.052 & 33.4 & 0.09 & 66.4 & 384 & 9.1 \\
\hline 1200_2 & 1065 & 480 & 0.068 & 42.8 & 0.09 & 79.1 & 609 & 9.5 \\
\hline 1200_3 & 1065 & 810 & 0.110 & 45.7 & 0.11 & 77.4 & 438 & 15.0 \\
\hline 1500_3 & 1483 & 867 & 0.087 & 50.3 & 0.13 & 85.9 & 546 & 14.6 \\
\hline $1800 \_3^{9)}$ & 1765 & 780 & 0.067 & I & I & 122.3 & I & I \\
\hline
\end{tabular}

${ }^{1)}$ All samples had degree of polymerization (DP) of the side chains of bottlebrush block $n_{\mathrm{sc}}=14$ separated by $n_{\mathrm{g}}=1$. DPs of the backbone of the bottlebrush block ${ }^{2)}$ and the PMMA linear block ${ }^{3)}$ were determined by ${ }^{1} \mathrm{H}-\mathrm{NMR}$ within a standard deviation of 5\% and 2\%, respectively (Figures S1 and S2), as well as with AFM (Figures S3A, S3B and Table S1). ${ }^{4}$ Volume fraction of PMMA block is determined from chemical composition of ABA bottlebrushes. ${ }^{5)}$ Diameter of PMMA spherical domains is determined from the form-factor of spheres (Figure 2). ${ }^{6}$ Relative standard deviation of $\mathrm{D}_{\mathrm{A}} \cdot{ }^{7)}$ Position of the main interference 
peak (see text for details). ${ }^{8)}$ Aggregation number determined as $Q=\pi D_{A}^{3} /\left(6 n_{A} v_{A}\right)$, where $v_{A}=0.141 \mathrm{~nm}^{3}$ is volume of methyl methacrylate. ${ }^{9)}$ Area per bottlebrush strand at the interface with PMMA spherical domains.

The significant difference in the solubility parameter between PMMA and PDMS and correspondingly large value of the Flory-Huggins interaction parameter $(\chi=0.2)$ results in strong segregation between the PMMA and PDMS blocks. [5] The PMMA-blocks (A) form spherical domains linked together by PDMS bottlebrush strands (B) (Figure 1b). This arrangement close to the interface may be viewed as a brush-on-brush system, where side chains are grafted to the bottlebrush backbone, which in turn is anchored at the A-B interface (Figure 1c).

Selected series of USAXS-SAXS curves are shown in Figure 2. The left panel displays scattering curves that correspond to samples with the same bottlebrush block $\left(n_{b b}=\right.$ 938) and variable length of the terminal PMMA blocks $\left(n_{A}=190,325,656\right.$, and 1235). The right panel shows curves corresponding to ABA samples with an approximate $n_{A}$ of the PMMA block and variable $n_{b b}$ of the PDMS bottlebrush block. These curves exhibit several characteristic features: (i) a strong interference maximum in the USAXS region corresponding to the spatial arrangement of the PMMA spheres $\left(d_{3}\right)$, (ii) a series of ripples $\left(d_{2}\right)$ that correspond to the form-factor of the PMMA spheres at higher angles, and (iii) a single peak $\left(d_{1}\right)$ due to scattering from bottlebrushes caused by the electron-density contrast between the brush backbone and that of the side chains.

To verify the assignments of $d_{1}$ and $d_{2}$, we monitored the variation of the peaks positions upon swelling in different solvents. The $d_{1}$ peak consistently shifts to smaller angles when swollen in PDMS-selective solvents such as $n$-hexane and linear PDMS oligomers, however, its location remains unchanged when swollen in dimethylformamide a poor solvent for PDMS.(Figures S4.1 and S4.2). In contrast, the $d_{2}$-peak is not affected by swelling in PDMSselective solvents due to their immiscibility with PMMA. In addition, the intensity of the $d_{1}$-peak exhibits orientation during in-situ stretching, with the intensity maxima being located in the plane perpendicular to the stretching direction (Figure S5). Note that the $d_{1}$-peak was previously reported for polymer brushes with crystallizable side chains[9], bottlebrushes with $n$-butyl acrylate side chains [10], and $\alpha$-olefin bottlebrushes [11].

For quantitative analysis of the USAXS-SAXS curves, we present the scattering intensity $\mathrm{I}(\mathrm{q})$ as:

$$
I(q) \sim \mathrm{P}^{2}(q) S(q)
$$

where $S(q)$ is the structure factor and $P(q)$ is the formfactor of spherical domains which, for homogeneous monodisperse spheres, has the following form $P(q)=$ $3[\sin (q R)-q R \cos (q R)] /(q R)^{3}$. In eq $1 \mathrm{q}$ stands for the norm of the scattering vector defined as $|\mathbf{q}|=4 \pi \cdot \sin (\theta) / \lambda$, where $\theta$ is the Bragg angle and $\lambda$-wavelength. The polydispersity effect was incorporated by a convolution of eq 1 with the Gaussian size distribution functions. After extracting the PMMA sphere diameter $\left(\mathrm{D}_{\mathrm{A}}\right)$ and its polydispersity, the $S(q)$ functions were analyzed. The obtained $S(q)$ curves with a well-defined main interference maximum indicate that there is no long-range order in arrangement of the PMMA spheres. Therefore, the $S(q)$ curves were fitted to Kinning and Thomas model,[7] which accounts for both the hard-core [8] and soft interaction potentials in liquidlike systems. This fitting reproduces the lowest angle peak and in some cases the secondary peak (Figure S6). The obtained effective radius of the particles includes the hard sphere (i.e., PMMA), as well as a size of the surrounding soft shell.[7] The thickness of the covering layer varies between 7.7 and $16.8 \mathrm{~nm}$, which correlates with the length of the bottlebrush blocks. This analysis provides the inter-domain distance distribution as a function of the backbone DP $\left(n_{b b}\right)$. These results are also consistent with AFM observations (Figures S3A,B).

Evaluation of the local backbone conformation was performed by using the well-defined form-factor $P(q)$ and the brush peak $\left(d_{1}\right)$. Position of the $d_{1}$-peak corresponding to the backbone-to-backbone separation reflects packing of bottlebrushes in the melt that fills the space between the PMMA domains far from the A-B interface. Due to strong steric repulsion, side chains do not overlap and bottlebrushes behave as filaments with a persistence length on the order of their diameter. $[1,12,13]$ We can use average distance between backbones $d_{1}=3.4 \mathrm{~nm}$ to estimate the local backbone conformation inside the bottlebrush envelope. Indeed, from distance $d_{1}$ the average area per side chain is equal to $S_{\text {bulk }} \cong \sqrt{3} d_{1}^{2} / 2 \cong 10 \mathrm{~nm}^{2}$. Using the volume $V_{s c}=M_{s c} /\left(N_{A v} \rho\right)=1.73 \mathrm{~nm}^{3}$ occupied by a side chain with a molar mass of the side chain $M_{s c}=$ $1024 \mathrm{~g} / \mathrm{mol}$ and density of bottlebrush $\rho=$ $0.97 \mathrm{~g} / \mathrm{cm}^{3}$, the distance between neighboring side chains along the bottlebrush backbone is estimated as $l_{\text {bulk }}=V_{s c} / S_{\text {bulk }}=0.17 \mathrm{~nm}$. Note that $l_{\text {bulk }}$ is smaller than the backbone monomer projection length $l_{0}=$ $0.25 \mathrm{~nm}$. This points out to a moderate extension of the backbone inside the bottlebrush envelope.

The spatial organization of the bottlebrush blocks at the AB interface can be obtained by approximating scattering of PMMA domains by the form-factor of polydisperse solid spheres (see Table 1 for sphere diameter $\left(D_{A}\right)$ and its dispersity). This directly gives the aggregation number $Q=$ $\pi D_{A}^{3} /\left(6 v_{A} n_{A}\right)$ and the average area per molecule $\left(S_{\text {interf }}\right)$ at the surface of the PMMA domains as:

$$
S_{\text {interf }}=\frac{\pi D_{A}^{2}}{Q}=\frac{6 v_{A} n_{A}}{D_{A}}
$$

where $v_{A}=0.141 \mathrm{~nm}^{3}$ is the PMMA monomer volume. In the framework of the scaling approach based on the balance of the block's interfacial and stretching free energies, the diameter of PMMA spheres is related to the block's degrees of polymerization [3] as

$$
D_{A} \cong\left(n_{b b} n_{A} v_{A}\right)^{1 / 3}
$$


By substituting eq 2 into eq 3 , the following scaling relation for the molecular area at the A-B interface is estimated to be:

$$
S_{\text {interf }} \sim n_{A}^{2 / 3} n_{b b}^{-1 / 3}
$$

It can be seen that relationships (3) and (4) hold within ca. $18 \%$ precision for all of the studied systems (Figure 3).

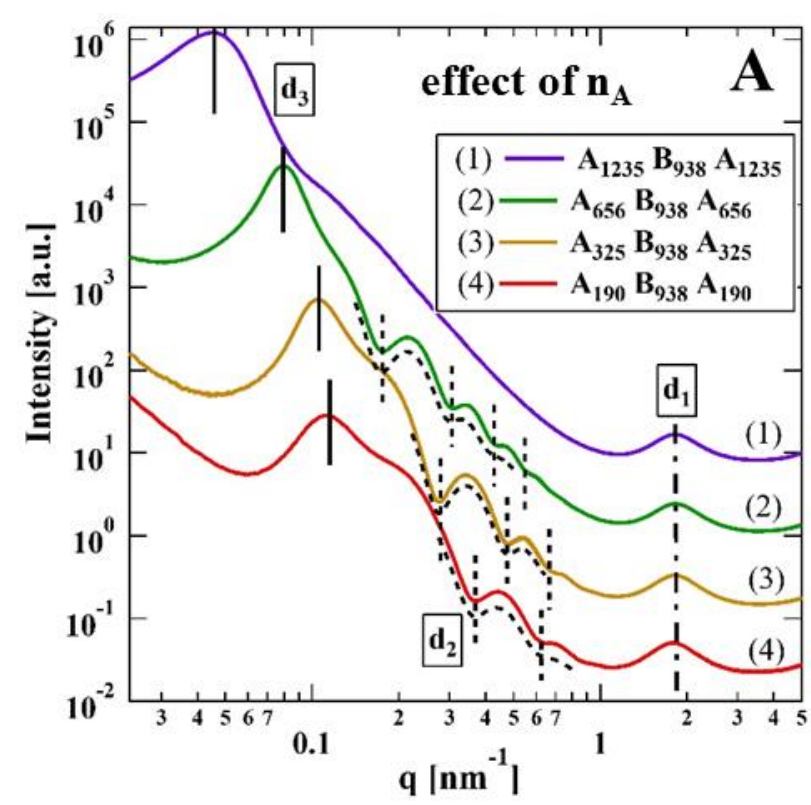

Note that the main source of error in this case is associated with uncertainty in the molecular parameters of the studied plastomers.

Figure 2. USAXS-SAXS curves corresponding to different series of ABA bottlebrush copolymer with (A) invariable B block and (B) approximately invariable $\mathbf{A}$ block. The fits with the form-factor of PMMA spheres are shown by the dashed lines. Note that the $\mathbf{A}_{\mathbf{1 2 3 5}} \mathbf{B} \mathbf{9 3 8} \mathbf{A}_{\mathbf{1 2 3 5}}$ sample with the longest A block does not display well-defined ripples of the spherical form-factor, which is ascribed to a transition to a non-spherical morphology as supported by AFM.[3]

Comparing the average area in the bulk with that at the AB interface, we can identify ABA systems with locally perturbed bottlebrush conformation. This is illustrated in Figure 3B by a dashed line $S_{\text {interf }} \sim S_{\text {bulk }}$. For ABAs with longer A-blocks and correspondingly larger domain sizes $D_{A}, S_{\text {interf }}$ approaches and then becomes bigger than the bulk value of $S_{\text {interf }} \sim S_{\text {bulk }} \approx 8.5 \div 15 \mathrm{~nm}^{2}$. However, systems with shorter A-blocks (smaller domains with higher curvature) demonstrate progressive decrease of the bottlebrush footprint at the A-B interface down to $4.1 \mathrm{~nm}^{2}$ (Table 1). This results in additional local stretching of the bottlebrush block including stretching and alignment of side chains along the backbone.

We can estimate how far from the interface the deformation of the bottlebrush block should persist. To do so, we introduce a dimensionless crowding parameter $\Phi$ - ratio of the volume occupied by sections of the bottlebrush block $V_{b b}(\Delta)$ within a spherical shell of thickness $\Delta$ around A domains to the volume of the spherical shell $V_{s h}(\Delta)$. In our analysis of the bottlebrush packing in the vicinity of the A domains, we assumed that the bottlebrush backbones are aligned in radial direction to reduce their footprint (Figure 1C). The volume occupied by bottlebrushes is equal to $V_{b b}(\Delta)=Q V_{s c} \Delta / l_{0}$ (where $l_{0}=0.25 \mathrm{~nm}$ is the monomer projection length). Taking into account the explicit expression for the volume of the spherical shell and substituting for the aggregation number $Q=4 \pi R_{A}^{3} /\left(3 v_{A} n_{A}\right)$ in terms of the spherical domain size, $R_{A}$, we obtain:

$$
\Phi(\Delta)=\frac{R_{A}^{3}}{n_{A} l_{0}} \frac{\mathrm{v}_{s c}}{v_{A}}\left(3 R_{A}^{2}+3 R_{A} \Delta+\Delta^{2}\right)^{-1}
$$

The value of the crowding parameter $\Phi>1$ indicates that all monomers belonging to the side chains cannot be packed within a spherical shell and should be expelled. The value of the crowding parameter at the surface of the spherical domain $\Phi(0)$ is larger than unity only for 300 -x series and one 900-1 data set (see Table S3). Thus, for these systems, packing of the bottlebrushes close to A-B interface could require stretching of the backbone and stretching and alignment of the side chains along the bottlebrush backbone. Such additional straining of the bottlebrush as compared to the conformation in the bulk will continue until $\Phi \approx 1$. The thickness of the spherical shell $\Delta^{*}$ at crossover to the bulk-like behavior is obtained by solving eq 5 for $\Delta$ with crowding parameter $\Phi=1$. We can extend this analysis to the case when backbones maintain their original bulk conformations close to interface and side chains are aligned along the direction normal to the interface. In this case we substitute $l_{\text {bulk }}=0.17 \mathrm{~nm}$ instead of the monomer projection length $l_{0}$ in eq 5 . The results of these calculations are summarized in Table S3. We would like to point out that direct probing of the brush conformation at 
interface could be obtained from neutron scattering experiments, [14] unfortunately, precise deuteration of the bottlebrushes remains a challenging synthetic task.

In conclusion, the combination of USAXS and SAXS techniques highlights the hierarchical organization of linearbottlebrush-linear PMMA-PDMS-PMMA triblock copolymer physical networks. A new feature is the dependence of bottlebrush conformation on the distance from the PMMA-PDMS A-B interface and the interface curvature. Close to the interface with a diameter of $D_{A}<27 \mathrm{~nm}$, the backbones are aligned in the radial direction while side chains are stretched and aligned with the backbones in order to satisfy local packing constraints. (Figure 1C). Far from the interface, bottlebrushes behave as flexible filaments with moderately extended backbones. A similar conformational behavior on both sides of the A-B interface should also be expected for other types of block-copolymer with brush-like blocks [15-17]. We hope our results will inspire investigation of bottlebrush conformation in such systems.

\section{ASSOCIATED CONTENT}

The Supporting Information is available free of charge on the ACS Publications website.

Materials and synthesis, NMR characterization of the bottlebrush copolymers, molecular and structural parameters of the studied bottlebrush copolymers, AFM characterization, SAXS data treatment and analysis, structure evolution of the bottlebrush copolymers upon swelling in selective solvents, orientation of the brush
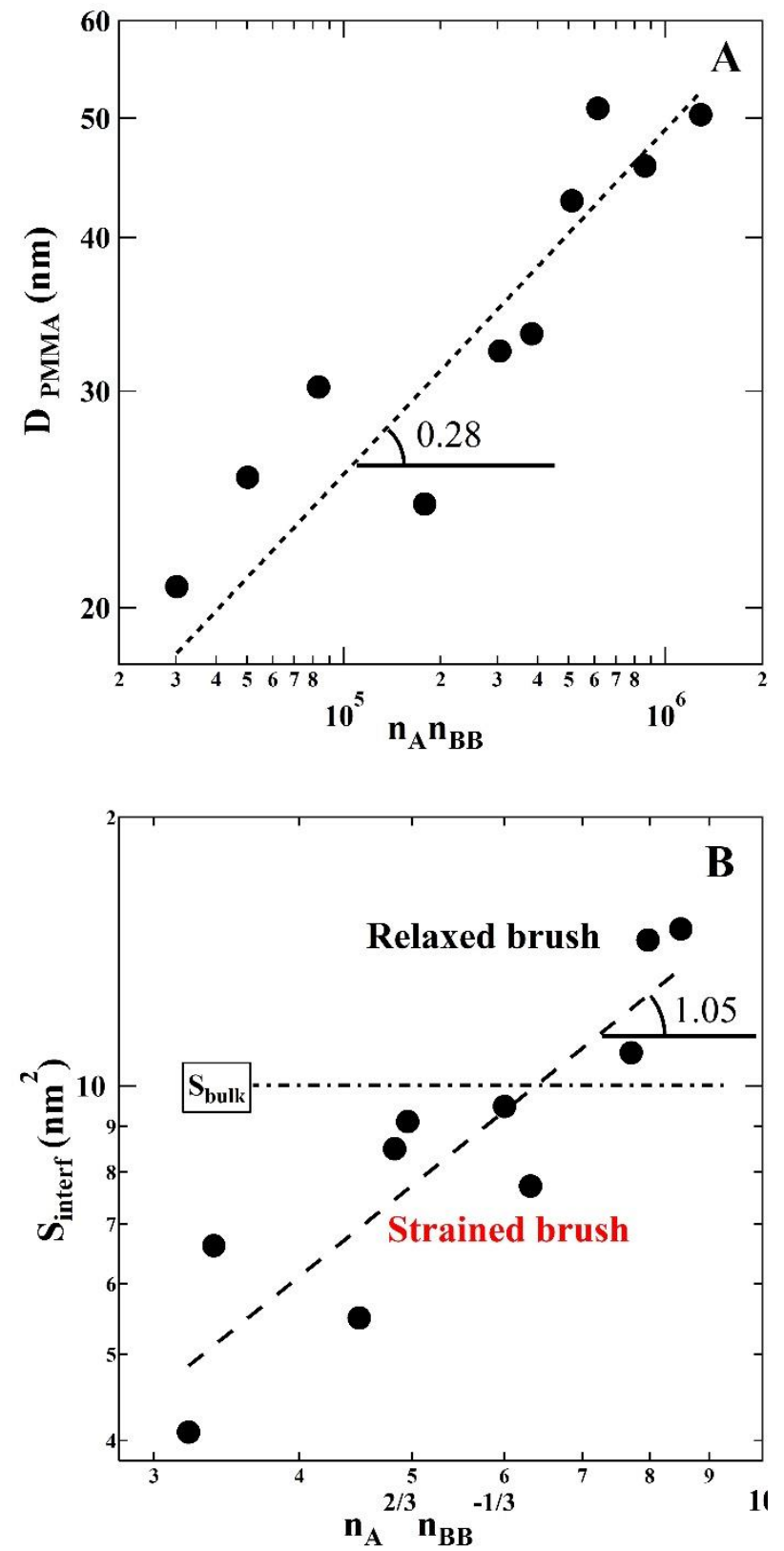

Figure 3. (A) Scaling dependences for the PMMA domain diameter $D_{A}$ and (B) the molecular area at the A-B interface $S_{\text {interf }}$ as a function of degrees of polymerization of the PMMA linear block $\left(n_{A}\right)$ and PDMS bottlebrush backbone $\left(n_{b b}\right)$.

peak upon stretching, fitting of the structure factor with the Kinning-Thomas model, and analysis of packing of the bottlebrushes (PDF).

\section{AUTHOR INFORMATION}

\section{Corresponding Author}

*Email: dimitri.ivanov@uha.fr.

\section{Funding Sources}

USAXS and SAXS experiments were supported by the Russian Science Foundation (Contract No. 16-13-10369). This work was performed in accordance with the state task No. 0074-2019-0014. 
S.S.S and A.V.D. gratefully acknowledge funding from the National Science Foundation (DMR 1407645, DMR 1436201, DMR 1436219, and DMR 1624569) and from the Ministry of Education and Science of the Russian Federation within State Contract N 14.W03.31.0022.

\section{ACKNOWLEDGMENT}

USAXS-SAXS measurements were performed at the ID02 beamline, European Synchrotron Radiation Facility (ESRF). The authors acknowledge perfect technical support during the measurements at the ESRF.

\section{REFERENCES}

(1) Daniel, W.F.M.; Burdyńska, J.; Vatankhah-Varnoosfaderani, M.; Matyjaszewski, K.; Paturej, J.; Rubinstein, M.; Dobrynin, A.V.; Sheiko, S.S. Solvent-Free, Supersoft and Superelastic Bottlebrush Melts and Networks. Nature Materials 2016, 15, 183-189.

(2) Vatankhah-Varnosfaderani, M.; Daniel, W.F.M..; Zhushma, A.P.; Li Q.; Morgan, B. J.; Matyjaszewski, K.; Armstrong, D.P.; Spontak, R.J.; Dobrynin,A.V.; Sheiko, S.S. Mimicking Biological Stress-Strain Behaviour with Synthetic Elastomers, Nature 2017, 549, 497-501.

(3) Vatankhah-Varnosfaderani, M.; Keith, A.N.; Cong, Y.; Liang, H.; Rosenthal, M.; Sztucki, M.; Clair, C.; Magonov, S.; Ivanov, D.A.; Dobrynin, A.V.; Sheiko, S.S. Chameleonlike Elastomers with Molecularly Encoded Strain-Adaptive Stiffening and Coloration Science 2018, 359, 1509-1513.

(4) Bates, F. S.; Fredrickson, G. H. Conformational Asymmetry and Polymer-Polymer Thermodynamics. Macromolecules 1994, 27 (4), 1065-1067.

(5) Luo, Y.; Montarnal, D.; Kim, S.; Shi W.; Barteau, K.P.; Pester, C.W.; Hustad, P.D.; Christianson, M.D.; Fredrickson, G.H.; Kramer, E.J.; Hawker, C.J. Poly(dimethylsiloxane- $b^{-}$ methyl methacrylate): A Promising Candidate for Sub-10 nm Patterning. Macromolecules 2015, 48, 3422-3430.

(6) Liang, H.; Morgan, B.J.; Xie, G.; Martinez, M.R.; Zhulina, E.B.; Matyjaszewski, K.; Sheiko, S.S.; Dobrynin, A.V. Universality of the Entanglement Plateau Modulus of Comb and
Bottlebrush Polymer Melts. Macromolecules 2018, 51, 10028-10039.

(7) Kinning, D.J.; Thomas, E.L. Hard-Sphere Interactions between Spherical Domains in Diblock Copolymers. Macromolecules 1984, 17, 1712-1718.

(8) Percus, J. K.; Yevick, G. J. Analysis of Classical Statistical Mechanics by Means of Collective Coordinates. Phys. Rev. 1958, 110, 1-13.

(9) Yu-Su, S.Y.; Sheiko, S.S.; Lee, H.-il; Jakubowski, W.; Nese, A.; Matyjaszewski, K.; Anokhin, D.; Ivanov, D.A. Crystallization of Molecular Brushes with Block Copolymer Side Chains. Macromolecules 2009, 42, 9008-9017.

(10) Pakula, T.; Minkin, P.; L. Beers, K.; Matyjaszewski, K. Structure and Dynamics in Melts of Bottle-Brush Polymers. Polymeric Materials: Science \& Engineering 2001, 84, 10061007.

(11) López-Barrón, C.R.; Tsou, A.H.; Younker, J.M.; Norman, A.I.; Schaefer, J.J.; Hagadorn, J.R.; Throckmorton, J.A. Microstructure of Crystallizable $\alpha$ - Olefin Molecular Bottlebrushes: Isotactic and Atactic Poly(1-octadecene). Macromolecules 2018, 51, 872-883.

(12) Cao, Z.; Carrillo, J.-M. Y.; Sheiko, S. S.; Dobrynin, A. V. Computer Simulations of Bottle Brushes: From Melts to Soft Networks. Macromolecules 2015, 48, 5006-5015.

(13) Liang, H.; Cao, Z.; Wang, Z.; Sheiko, S. S.; Dobrynin, A. V. Combs and Bottlebrushes in a Melt. Macromolecules 2017, 50, 3430-3437.

(14) Wei, Y.; Xu, Y.; Faraone, A.; Hore, M.J.A. Local Structure and Relaxation Dynamics in the Brush of Polymer-Grafted Silica Nanoparticles. ACS Macro Lett. 2018, 7, 699-704.

(15) Bates, C. M.; Bates, F. S. 50th Anniversary Perspective: Block Polymers: Pure Potential. Macromolecules 2017, 50 (1), 3-22.

(16) Dalsin, S. J.; Rions-Maehren, T. G.; Beam, M. D.; Bates, F. S.; Hillmyer, M. A.; Matsen, M. W. Bottlebrush block polymers: Quantitative theory and experiments. ACS Nano 2015, 9 (12), 12233-12245.

(17) Gu, W.; Huh, J.; Hong, S. W.; Sveinbjornsson, B. R.; Park, C.; Grubbs, R. H.; Russell, T. P. Self-assembly of symmetric brush diblock copolymers. ACS Nano 2013, 7 (3), 25512558 . 
${ }^{8}$ Moscow Institute of Physics and Technology (State University), Institutskiy per. 9, Dolgoprudny, Russian Federation;

${ }^{9}$ Institute of Problems of Chemical Physics, Russian Academy of Sciences, Chernogolovka, Moscow region, 142432, Russian Federation.

\section{Strained Bottlebrushes in Su- per-Soft Physical Networks}

Charles Clair, ${ }^{1}$ Abdelaziz Lallam, ${ }^{1}$ Martin Rosenthal, ${ }^{2}$ Michael Sztucki, ${ }^{2}$ Mohammad Vatankhah-Varnosfaderani, ${ }^{3}$ Andrew N. Keith, ${ }^{3}$ Yidan Cong, ${ }^{3}$ Heyi Liang, ${ }^{4}$ Andrey V. Dobrynin, ${ }^{4}$ Sergei S. Sheiko, ${ }^{3,5}$ and Dimitri A. Ivanov $* 6,7,8,9$

${ }^{1}$ Laboratoire de Physique et Mécanique Textiles, F68093 Mulhouse Cedex, France;

${ }^{2}$ European Synchrotron Radiation Facility, F-38043 Grenoble, France;

${ }^{3}$ Department of Chemistry, University of North Carolina at Chapel Hill, Chapel Hill, NC 27599, USA;

${ }^{4}$ Department of Polymer Science, University of Akron, Akron, OH, 44325-3909, USA;

${ }^{5}$ Institute of Macromolecular Compounds of the Russian Academy of Sciences, St. Petersburg, 199004, Russian Federation;

${ }^{6}$ Institut de Sciences des Matériaux de Mulhouse-IS2M, CNRS UMR 7361, F-68057 Mulhouse, France;

${ }^{7}$ Lomonosov Moscow State University, Faculty of Fundamental Physical and Chemical Engineering, Leninskie Gory 1/51, 119991 Moscow, Russian Federation;

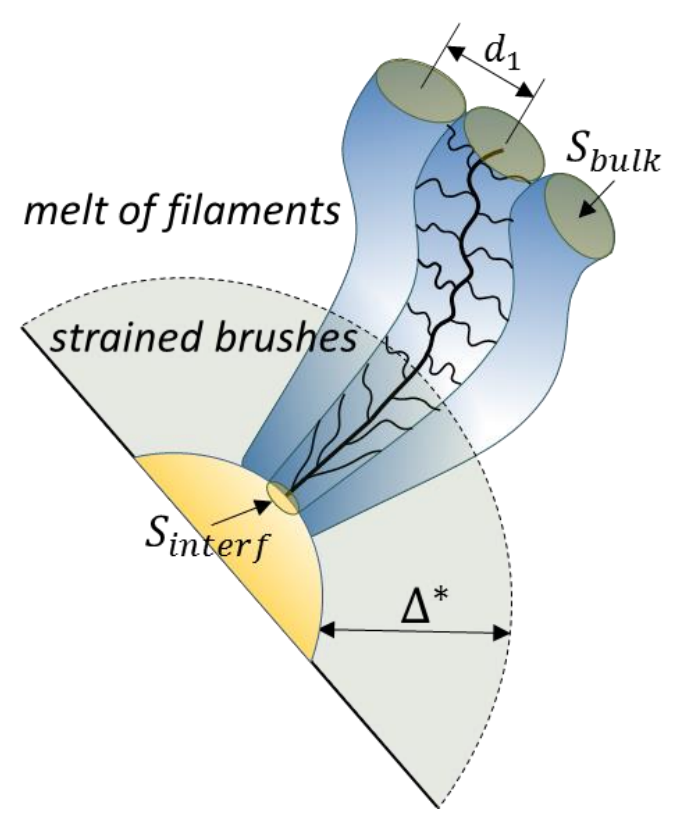

